\title{
Geometrical Interpretation of Time and New Models of the Space-Time
}

\author{
Oleg. A. Olkhov \\ Laboratory of Theoretical Physics, Institute of Chemical Physics of Russian Academy of Sciences, Moscow, Russia \\ Email: oleg.olkhov@rambler.ru
}

How to cite this paper: Olkhov, O.A. (2017) Geometrical Interpretation of Time and New Models of the Space-Time. Journal of High Energy Physics, Gravitation and Cosmology, 3, 564-571.

https://doi.org/10.4236/jhepgc.2017.34043

Received: July 17, 2017

Accepted: August 27, 2017

Published: August 30, 2017

Copyright $\odot 2017$ by author and Scientific Research Publishing Inc. This work is licensed under the Creative Commons Attribution International License (CC BY 4.0).

http://creativecommons.org/licenses/by/4.0/

\begin{abstract}
It is shown that two fundamental notions "space" and "time" can be reduced to the one notion-the "space", where time appears to be the length of geodesic lines of the space adjacent to the three-dimensional Euclidean space. The whole space is a topological product of the three-dimensional Euclidean space and some another space, that may have the screw structure. Within the framework of this model, the Galilei's inertia law and existence of limiting velocity of motions are consequences of the geometrical interpretation of time.
\end{abstract}

\section{Keywords}

Special Relativity, Geometrization of Physics, Geometrical Paradigm, Geometrization of Time

\section{Introduction}

Space and time are fundamental physical notions, and they are basis for definition of the central notion of natural sciences-the notion of "motion". It is impossible to express these notions in terms of more fundamental ones. The time is considered in special relativity as one of the four coordinates $\left(t, x_{1}, x_{2}, x_{3}\right)$ in the four-dimensional so called "space of events", where scalar $t$ is the "moment of time", when the event takes place, and $x_{1}, x_{2}, x_{3}$ are coordinates of the "place of event" [1] [2] [3]. Hard sticks are standards for measuring of coordinates of "places of events" and periodical movements (clocks) are standards for measuring of "moments of time". Definition of standards means that the space of event can be investigated by physical methods, and it was established that three coordinates $x_{1}, x_{2}, x_{3}$ describe a three-dimensional Euclidean space. This space looks, intuitively, as understandable one because its standards have dimensionality of length. On the other hand, time does not look understandable on the basis of 
notions from everyday life. Here we show that time can be also considered as geometrical characteristic with dimensionality of length in some special many-dimensional space. It means that notions "space" and "time" can be reduced to the one notion- "the space" with definite real geometry. Notice, that the suggestion about the same nature of the notions "space" and "time" was, may be, firstly proposed by Feynman [3].

This work is a continuation of author's investigations of possibility to explain physical phenomena by geometrical properties of the space-time [4] [5] [6] [7] [8]. In these investigations the idea was used about increasing dimensionality of our Euclidean space from three to four-the idea firstly used by Kaluza and then by Bergmann and Einstein in their attempts to geometrize electromagnetic field [9] [10]. For geometrization of gauge fields this idea was later used by Vladimirov [11]. Ideas about additional space (additional to our Euclidean space) were also investigated by Urusovskii [12]. The idea about additional dimensionalities is the main idea of this work, and this idea is based on the hypothesis that the space of events is a special case of fiber spaces [2], namely, the topological product of the usual Euclidean space and some another space with real geometry. Preliminary results were earlier published in [13] [14]. Here we present the more rigorous consideration.

Notice, that the formal geometrical interpretation of time is known where time is considered as an imaginary fourth coordinate with the dimensionality of length in the four dimensional pseudo-Euclidean space that is often identified as a "space-time" (Minkowski space) [1]. But representation of time by means of imaginary numbers does not give an opportunity to understand reasons of deep difference between notions "space" and "time". Instead of the fictitious Minkowski space, the geometrical models of the space-time are considered, where all coordinates are real numbers.

\section{Euclidean Model}

Geodesic lines in the space define the shortest distances between any two points and for the space with the connectivity $\Gamma_{i j}^{k}$ they are described in the Riemannian space by the known Equations [2]

$$
\frac{\mathrm{d}^{2} x^{k}}{\mathrm{~d} \tau^{2}}=-\Gamma_{i j}^{k} \frac{\mathrm{d} x^{i}}{\mathrm{~d} \tau} \frac{\mathrm{d} x^{j}}{\mathrm{~d} \tau}, \Gamma_{i j}^{k}=g^{k l}\left(\frac{\mathrm{d} g_{i l}}{\mathrm{~d} x^{j}}+\frac{\mathrm{d} g_{j l}}{\mathrm{~d} x^{i}}-\frac{\mathrm{d} g_{i j}}{\mathrm{~d} x^{l}}\right), i, j=1,2, \cdots, n
$$

where $n$ is the dimensionality of the space, $\tau$ is a parameter, $g_{i k}$ is the metric of the space.

We start with the simplest possible model of the space-time $\left(M_{4}\right)$, when it is a topological product of our three-dimensional Euclidean space $R_{3}$ and one-dimensional Euclidean space $R_{1}: M_{4}=R_{3} \times R_{1}$. This resulting space is obviously the four-dimensional Euclidean space. Our main hypothesis is that the time of the body's motion with the definite velocity in our empty three-dimensional Euclidean space multiplied by light velocity equals to the length of the corresponding geodesic line in the space adjacent to our Euclidean space. In our case the 
adjacent space is also the Euclidean one. In Euclidean space all metric coefficients $g_{i k}$ are constants and all connectivities $\Gamma_{i j}^{k}$ equal to zero [2]. This means from (1) that all geodesics in our adjacent space are straight lines. At Figure 1 our three-dimensional Euclidean space is replaced (to be more descriptive) by the one-dimensional Euclidean space (axis $0 X$ ), so the adjacent space is a plane. Here $X$ is the displacement of the free body from the coordinate origin, $\alpha$ is an angel between geodesic lines and the direction of the body's displacement, $L$ is the length of geodesic lines corresponding to this displacement. Our main suggestion is that the length of geodesic lines $L$ is connected with the time $t$ of corresponding displacement $x$ by the relation

$$
c t=L .
$$

It follows from Figure 1

$$
x=L \cos \alpha=c t \cos \alpha=V t, \quad V=c \cos \alpha .
$$

So, our main suggestion (2) leads to the right law of the free body's motion in our Euclidean space-to the Galilei's inertia law, when the free body is moving with the constant velocity. And this proves suggested geometrical interpretation of time.

We see that within the framework of the suggested model the space of events is represented not by the fictitious four-dimensional pseudo-Euclidean Minkowski space, but it is represented by four-dimensional Euclidean space, where time has the special geometrical interpretation. Notice that suggested interpretation of time gives the opportunity for simple explanation of the existence of limiting velocity of any motion. Indeed, because $\cos \alpha \leq 1$ we obtain from (3)

$$
V \leq c \text {. }
$$

For limiting velocity of motions $\cos \alpha=1$, and we obtain from (3)

$$
x=L \text { or } x=c t \text {. }
$$

We obtain this relation for the one-dimensional model of our three-dimensional Euclidean space. For real three-dimensional space relation (5) is obviously generalized as

$$
x^{2}+y^{2}+z^{2}=L^{2}=c^{2} t^{2}
$$

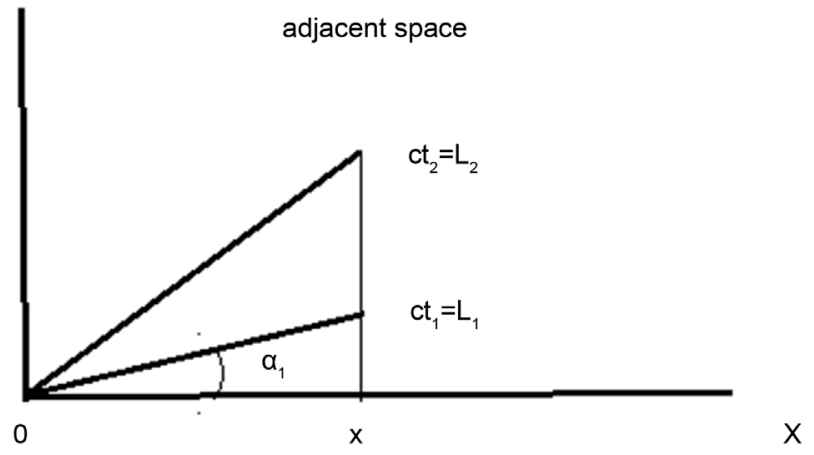

Figure 1. Time as a length of geodesic lines. 
This relation is one of the basic relations of special relativity [1].

\section{Non-Euclidean Model: Low-Dimensional Analogy}

In the preceding Section we considered Euclidean model of the space-time, and the space-time has here the simple symmetries of the Euclidean space. But there are some experimental facts, indicating that the space-time symmetry may be more complex. These are distortions of chiral symmetry in some native chemical compounds, non-conservation of parity in some interactions and distortion of charge symmetry (particle-antiparticle). In this Section we considered the model of the space-time with screw symmetry. To make the idea more understandable and descriptive we firstly consider the simplest possible low-dimensional analogy of the suggested model of the space-time that reflects important features (not all) of the suggested model of the space-time. Namely, we will consider an analogy where the three-dimensional Euclidean space is replaced by the one-dimensional one and where the adjacent space is a surface of the infinite cylinder with radius $R$. The one-dimensional space (straight line $X$ ) is a directrix for this cylinder (Figure 2). This two-dimensional space (we denote this space as $M_{2}$ ) is a topological product of the one-dimensional space $R_{1}$ and one-dimensional circle $S_{1}$, so $M_{2}=R_{1} \times S_{1}$.

The mathematics in this case is essentially simplified. First of all rewrite Equations (1), taking the length of geodesic $L$ as a parameter $\tau$, so for our case in cylindrical coordinates $x=x^{1}$ and angle $\alpha=x^{2}$ the equations take the form

$$
\frac{\mathrm{d}^{2} x}{\mathrm{~d} L^{2}}=-\Gamma_{i j}^{x} \frac{\mathrm{d} x^{i}}{\mathrm{~d} L} \frac{\mathrm{d} x^{j}}{\mathrm{~d} L}, \frac{\mathrm{d}^{2} \alpha}{\mathrm{d} L^{2}}=-\Gamma_{i j}^{\alpha} \frac{\mathrm{d} x^{i}}{\mathrm{~d} L} \frac{\mathrm{d} x^{j}}{\mathrm{~d} L}, i, j=x, \alpha
$$

Let us firstly show that geodesic lines of this space are screw lines. Metric $g_{i k}$ is defined for Riemannian space by relation for an element of length $\mathrm{d} l[2]$

$$
\mathrm{d} l^{2}=g_{i k} \mathrm{~d} x^{i} \mathrm{~d} x^{k}
$$

For cylindrical surface with radius $R$ we have in terms of cylindrical coordinates $x$ and angle $\alpha$

$$
\mathrm{d} l^{2}=\mathrm{d} x^{2}+R^{2} \mathrm{~d} \alpha^{2} .
$$

From $(7,8)$, we have for nonzero coordinates of $g_{i k}$ of our cylindrical space

$$
g_{x x}=1, g_{\alpha \alpha}=R^{2}
$$

Such metric is called locally Euclidean one. Inserting (10) into $(1,7)$, we see

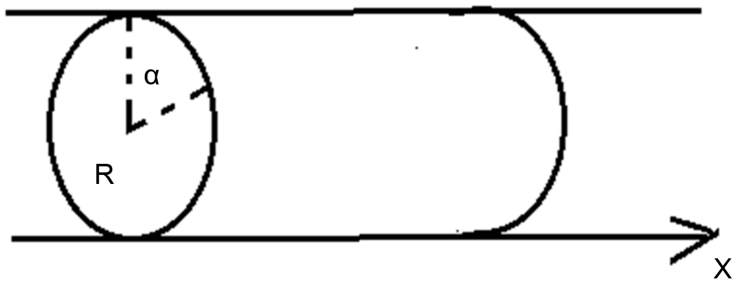

Figure 2. Cylindrical adjacent space. 
that right sides in Equations (7) equal to zero. This means that equations for $x$ and $\alpha$ have a simple form

$$
\frac{\mathrm{d}^{2} x}{\mathrm{~d} L^{2}}=0, \frac{\mathrm{d}^{2} \alpha}{\mathrm{d} L^{2}}=0
$$

The solution of these equations has the form

$$
x=C_{1} L, \alpha=C_{2} L,
$$

where $C_{1}$ and $C_{2}$ are constants. This solution describes geodesic screw lines on the cylinder. The constant $C_{1}$ is defined by the condition $x=h$ at $L=l$, where $h$ is a pitch of screw and $l$ is a length of the one turn of the screw line. This gives

$$
x=\frac{h}{l} L .
$$

There is a known relation for the crew line with radius $R$

$$
l^{2}=h^{2}+(2 \pi R)^{2} .
$$

From $(13,14)$, we have

$$
x=\frac{h}{\sqrt{h^{2}+(2 \pi R)^{2}}} L .
$$

According to our geometrical interpretation of time, the time $t$ of the free body's displacement in Euclidean space at the distance $x$ is defined by the relation

$$
c t=L .
$$

Inserting (16) in (15), we see that our geometrical interpretation of time (16) leads to the right law of free body's motion to the Galilei's inertia law of motion with the constant velocity

$$
x=V t, \quad V=\frac{h c}{\sqrt{h^{2}+(2 \pi R)^{2}}} .
$$

As for Euclidean model (Section 2), the suggested interpretation explains also the existing of limiting velocity of motions that equals to light velocity. Indeed, it follows from (17)

$$
V \leq c .
$$

This result has a simple geometrical explanation: the length of screw line cannot be larger than the corresponding straight line.

\section{Non-Euclidean Model: Five-Dimensional Space-Time}

We consider here the general model of the space-time with the screw structure. Namely, we consider the five-dimensional space-time $M_{5}$ when its structure is defined as topological product of our three-dimensional Euclidean space and a two-dimensional sphere:

$$
M_{5}=R_{3} \times S_{2}
$$


For better representation of such space we showed at Figure 3 the more simple example of analogous topological product $M_{4}=R_{2} \times S_{2}$, where Euclidean space is the two-dimensional one and where the hole space is embedded into four-dimensional space. In four-dimensional space two-dimensional spheres can be tightly embedded one into another as it is shown schematically at Figure 3. The situation here is analogous to the one in Section 2. with $M_{2}=R_{1} \times S_{1}$, where one-dimensional circles can form a two-dimensional cylindrical surface only in three-dimensional space.

Let us define now the form of geodesic lines in the space $M_{5}$. For five-dimensional space $M_{5}=R_{3} \times S_{2}$ Equations (1) will be the system of five nonlinear differential equations with $5^{3}=125$ coefficients of connectivity $\Gamma_{i j}^{k}$, but most of them equal to zero in our case. The five-dimensional space $M_{5}$ is a Riemannian one, where at every point the three-dimensional Euclidean space is perpendicular to the two-dimensional sphere. So the element of length in this five-dimensional space is defined by the relation

$$
\mathrm{d} l^{2}=\mathrm{d} x^{2}+\mathrm{dy} y^{2}+\mathrm{d} z^{2}+R^{2} \mathrm{~d} \theta^{2}+R^{2} \sin \theta^{2} \mathrm{~d} \varphi^{2}
$$

Here $x, y, z$ are Cartesian coordinates in Euclidean space and $\theta, \varphi$ are spherical coordinates on the sphere with radius $R$. This relation gives the following nonzero components of metric tensor $g_{i k}$

$$
g_{x x}=g_{y y}=g_{z z}=1, g_{\theta \theta}=R^{2}, g_{\varphi \varphi}=R^{2} \sin \theta^{2} .
$$

Inserting these values in (1), we obtain for nonzero components of $\Gamma_{i j}^{k}$

$$
\Gamma_{\theta \varphi}^{\varphi}=\Gamma_{\varphi \theta}^{\varphi}=\cot \theta, \Gamma_{\varphi \varphi}^{\theta}=-\sin \theta \cos \theta .
$$

Inserting this in (10) and taking the length of geodesic $L$ as a parameter $\tau$, we obtain

$$
\begin{gathered}
\frac{\mathrm{d}^{2} x}{\mathrm{~d} L^{2}}=0, \frac{\mathrm{d}^{2} y}{\mathrm{~d} L^{2}}=0, \frac{\mathrm{d}^{2} z}{\mathrm{~d} L^{2}}=0, \\
\frac{\mathrm{d}^{2} \theta}{\mathrm{d} L^{2}}=\sin \theta \cos \theta\left(\frac{\mathrm{d} \varphi}{\mathrm{d} L}\right)^{2}, \frac{\mathrm{d}^{2} \varphi}{\mathrm{d} L^{2}}=-2 \tan \theta \frac{\mathrm{d} \theta}{\mathrm{d} L} \frac{\mathrm{d} \varphi}{\mathrm{d} L} .
\end{gathered}
$$

These equations describe complex screw lines in the five-dimensional space. We choose the simple solution, analogous to the one in Section 2, when the screw line has the form of the screw line with the constant radius $R$. Namely,

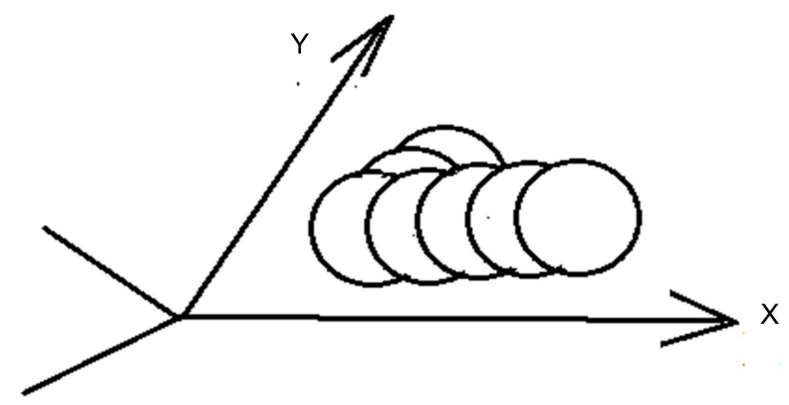

Figure 3. Topological product $M_{4}=R_{2} \times S_{2}$. 
we take the solution

$$
x=C_{1} L, y=C_{2} L, z=C_{3} l, \theta=\text { constant }=0, \varphi=C_{4} L .
$$

As in Section (2) constants $C_{1}, C_{2}, C_{3}$ are defined be the condition that $L=l$ for the displacement along the screw line (along the free body's displacement) at distance $\boldsymbol{h}$ (here $\boldsymbol{h}$ is a pitch of screw, $l$ is a length of screw line for one turn). This gives

$$
x=\frac{h_{x}}{l} L, y=\frac{h_{y}}{l} L, z=\frac{h_{z}}{l} L,
$$

where $h_{x}^{2}+h_{y}^{2}+h_{z}^{2}=h^{2}$. As in Section 2 we use now the relation, connecting $l, h$ and $R$ for a screw line

$$
l^{2}=h^{2}+(2 \pi R)^{2} .
$$

As a result, we have from $(25,26)$

$$
x=\frac{h_{x}}{\sqrt{h^{2}+(2 \pi R)^{2}}} L, y=\frac{h_{y}}{\sqrt{h^{2}+(2 \pi R)^{2}}} L, z=\frac{h_{z}}{\sqrt{h^{2}+(2 \pi R)^{2}}} L .
$$

According to our geometrical interpretation of time, the time $t$ of the free body's displacement in Euclidean space at the distance $\sqrt{x^{2}+y^{2}+z^{2}}$ is defined by the relation

$$
c t=L .
$$

Inserting (28) in (27), we see that our geometrical interpretation of time (28) leads to the right law of free body's motion-to the Galilei's inertia law of motion with the constant velocity

$$
x=V_{x} t, V_{x}=\frac{h_{x} c}{\sqrt{h^{2}+(2 \pi R)^{2}}}, y=V_{y} t, V_{y}=\frac{h_{y} c}{\sqrt{h^{2}+(2 \pi R)^{2}}}, z=V_{z} t, V_{z}=\frac{h_{z} c}{\sqrt{h^{2}+(2 \pi R)^{2}}} .
$$

As for Euclidean model (Section 2), the suggested interpretation explains also the existing of limiting velocity of motions that equals to light velocity. Indeed, it follows from (29)

$$
V \leq c .
$$

This result has again a simple geometrical explanation: the length of screw line cannot be larger than the corresponding straight line. In limiting case

$$
L=\sqrt{x^{2}+y^{2}+z^{2}}, c t=\sqrt{x^{2}+y^{2}+z^{2}}, c^{2} t^{2}=x^{2}+y^{2}+z^{2} .
$$

The last relation is the one of the basic relations of special relativity [1].

\section{Conclusions}

The geometrical interpretation of time is suggested where time of the free body's displacement multiplied by light velocity equals to the length of the geodesic line in the space adjacent to the Euclidean space. The models of the space-time are considered where above adjacent space is the Euclidean one or the space with the screw structure. The suggested models give the geometrical explanation for 
the Galilei's inertia law and for existence of the limiting velocity of any motion.

Within the framework of the suggested interpretation, time is intimately connected with the motion, and any kind of motions can be selected as the standard for the time measurements (periodical movements appeared to be the most convenient). No motion, no time. If the observer is immovable, then the time is connected with the movements in the clocks. If there are no clocks nearby, then the time is connected with the movements inside the observer.

\section{References}

[1] Pauli, W. (1958) Theory of Relativity. Pergamon Press, Oxford.

[2] Dubrovin, B.A., Fomenko, A.T. and Novikov, S.P. (1985) Modern Geometry-Methods and Applications. Springer, Berlin.

[3] Feynman, R., Leighton, R. and Sands, M. (1963) The Feynman Lectures on Physics. Addison-Wesley Publishing Company, Boston.

[4] Olkhov, O.A. (2007) Geometrization of Quantum Mechanics. Journal of Physics. Conference Series, 67, 012037-012041. https://doi.org/10.1088/1742-6596/67/1/012037

[5] Olkhov, O.A. (2007) Geometrization of Matter Wave Fields and Electromagnetic Waves. Proc. of X111 Int. Scientific Meeting PIRT-2007, Moscow, 2-5 July, 318.

[6] Olkhov, O.A. (2008) Geometrization of Classical Wave Fields. AIP Conferences Proceedings, 962, 316-321. https://doi.org/10.1063/1.2827325

[7] Olkhov, O.A. (2008) On the Possibility of Topological Interpretation of Quantum Mechanics. http://arXiv.org/abs/0802.2269

[8] Olkhov, O.A. (2014) Geometrical Approach in the Atomic Spectra Theory. Russian Journal of Physical Chemistry B, 8, 30-42. https://doi.org/10.1134/S199079311401014X

[9] Kaluza, Th. (1921) On the Unification Problem in Physics. Sitzungsberichte Preuss. Acad. Sci, 966-972.

[10] Einstein, A. and Bergmann, P. (1938) On a Generalization of Kaluza's Theory of Electricity. Annals of Mathematics, 39, 685. https://doi.org/10.2307/1968642

[11] Vladimirov, Yu.S. (2005) Geometrophysics (In Russian). BINOM, Laboratory of Knowledge, Moscow.

[12] Urusovskii, I.A. (2014) Multidimensional Treatment of the Expanding Universe. International Journal of Physical Sciences, 4, 1110. https://doi.org/10.9734/PSIJ/2014/10270

[13] Olkhov, O.A. (2017) On Possibility of Geometrical Interpretation of Time. Journal of High Energy Physics, Gravitation and Cosmology, 3, 173. https://doi.org/10.4236/jhepgc.2017.32018

[14] Olkhov, O.A. (2017) Geometrical Interpretation of Time. Proceedings of International Conference PIRT-17, Physical Interpretation of Relativity Theory, Moscow, 3-6 July 2017. (In press)

[15] Rashewski, P.K. (1958) Differential Geometry (in Russian). Nauka, Moscow. 